\title{
Sib competition in Tribolium: a test of the elbow-room model
}

\author{
CARLOS GARCIA* \& MIGUEL A. TORO† \\ Area de Xenética, Departamento de Bioloxia Fundamental, Facultade de Bioloxia, Santiago de Compostela, Galicia, \\ Spain and $\dagger$ Departamento de Producción Animal, CIT-INIA, Carretera de la Coruña, $\mathrm{Km} 7 s / n$, Madrid, Spain
}

\begin{abstract}
The elbow-room model of sib competition predicts that sexually reproducing parents could have a fitness advantage over asexual parents if competition within more diverse, sexually produced offspring was less intense than within more homogeneous, asexually produced ones. To test the assumption empirically that genetic homogeneity implies stronger competition, we compared the productivity of sib groups and random groups of the flour beetle Tribolium castaneum maintained at very high densities. For larval production, we found an initial advantage of sib groups but it did not result in an advantage in total adult production, because high initial densities increased mortality in the long run, such that the adult production was the same in both groups. We discuss how some changes in our experimental design could produce very different results, and conclude that sib competition is unlikely to be a general explanation for the widespread presence of sexual reproduction in nature.
\end{abstract}

Keywords: density-dependent viability, pupation inhibition, sexual reproduction.

\section{Introduction}

Two different theoretical models include sib competition as a mechanism to account for the widespread presence of genetic recombination and sexual reproduction in nature (Young, 1981). Both models rely on the advantage of increasing genetic variability in the offspring. In the 'lottery model', the environment is divided into spatial patches, each colonized by different sib families. If a patch provides resources for the development of just a few of the colonizing individuals, only those genotypes closest to the optimum genotype in the patch would survive. Consequently, sexually reproducing parents would be at an advantage, because their offspring would be genetically more diverse, and therefore would have a greater probability of producing the best adapted genotype in a given patch (Williams \& Mitton, 1973). In the 'elbow-room model', the number of surviving individuals in a patch is not fixed, depending on competition after colonization. The intensity of competition between individuals increases with their genetic resemblance, resulting in similar ecological requirements. Therefore, the viability of a sib is frequency-dependent, and parents increase their fitness by increasing the genetic diversity in their offspring (Maynard Smith, 1978). This model

\footnotetext{
*To whom correspondence should be addressed.
}

depends on the assumption that distance in the genetic space can be mapped into distance in the ecological space (Young, 1981).

Some theoretical analyses of the lottery model have been carried out (Maynard Smith, 1978; Taylor, 1979; Bulmer, 1980; Barton \& Post, 1986). However, the elbow-room model is less tractable theoretically, because the outcome of competition and the resulting advantages of sexual and asexual reproduction depend on the details of selection, making it difficult to obtain general results (Barton \& Post, 1986). Notwithstanding, Price \& Waser (1982) developed a theoretical model including features of both the lottery model (the number of survivors in each patch is fixed) and the elbowroom model (fitness is frequency-dependent). In this case, sexuality can spread in a population if offspring dispersal is limited and rare genotypes are sufficiently favoured by selection. The last condition is related to the basic assumption of the elbow-room model, i.e. an increase in genetic variability may result in a decrease in competition intensity.

Some empirical tests of this assumption have been carried out. In them, the average value for some fitnessrelated character was compared between groups of individuals showing different degrees of genetic homogeneity. The experimental organisms used and the results obtained were diverse. Ellstrand \& Antonovics (1985) and Kelley et al. (1988) compared the 
average fitness in two types of groups (half-sibs or genetically identical individuals) of the grass Anthoxanthum odoratum grown in the field, and found superior averages for the less genetically homogenous groups. In contrast with the predictions of the elbowroom model, this superiority did not increase with planting density (Kelley, 1989a), and disappeared when the experiment was carried out in a greenhouse (Kelley 1989b).

In Drosophila melanogaster, Pérez-Tomé \& Toro (1982) found a disadvantage in adult production and developmental time in half-sib groups when compared with groups of randomly sampled individuals, this disadvantage being greater at higher culture densities (Martin et al. 1988). This is expected if the advantage was due to a reduced intensity of competition in the sib groups.

Other experiments failed to detect any relationship between genetic homogeneity and intensity of competition. In the grass Plantago lanceolata, Tonsor (1989) did not find significant differences between the average vegetative production and reproductive output of groups of non-related individuals, half-sibs, and fullsibs. Willson et al. (1987) studied the average growth of sib and non-sib groups in four plant species, finding little evidence of increased competition among sibs.

In Tribolium confusum, Jasienski et al. (1988) found that groups of sibs developed faster than groups of non-related individuals, but this was not the case in the related species $T$. castaneum. No significant differences in total production after competition were found in both species. Finally, a clear example of decreased competition in genetically homogeneous groups has been reported by Jasienski (1988), who observed a clear superiority in growth rate for Bombina variegata tadpoles when they were maintained in full-sib groups instead of groups of non-relatives.

It is clear that the outcome of sib competition must depend on a number of interacting ecological and behavioural factors, and the diversity illustrated by the outcome of the experiments listed above suggests that at least some of these factors are species dependent. These factors need not operate along the whole competition period, and their effects or interactions might vary with time. Therefore, they are better studied if the competition process is recorded along the whole of its duration, instead of simply evaluating its final results.

Using a Tribolium castaneum population, we have carried out an experiment in which individuals compete either in sib groups or in randomly sampled groups. We have recorded each group's production and composition at weekly intervals, so that the evolution of the sib competition process can be traced out.

\section{Materials and methods}

The Consejo laboratory population of Tribolium castaneum, collected in 1964 near Madrid, was used. The culture medium consisted of 95 per cent wholewheat flour and 5 per cent weight-dried brewer's yeast. Beetles were maintained at $32^{\circ} \mathrm{C}$ and 70 per cent relative humidity.

The experiment was initiated with two series of individuals, sampled as pupae in two consecutive days from the same big population cage. A series consisted of 50 mating groups, each formed by one male and 20 females, which were allowed to mate for 6 days. The eggs laid by these mating groups were recovered and used to set up competition vials, that contained $1 \mathrm{~g}$ of medium.

Two kinds of competition vials were set up: sib and random. In the sib competition vials, one sample of 100 eggs was taken from each mating group, and was transferred to a competition vial. Thus, every individual was surrounded by half and full sibs, and competition took place in genetically homogeneous groups. In the random competition vials, eggs laid by the females in one series were pooled, and samples of 100 eggs were randomly taken from the pool, each sample being transferred to a competition vial. Individuals growing in these vials were surrounded by genotypes randomly taken from the population, and competition took place in genetically heterogeneous groups.

The population density in both kinds of competition vials was therefore of 100 eggs $\mathrm{g}^{-1}$ of culture medium. This is a very high density in Tribolium, and was chosen because it was expected that any difference in production between the treatments should be easier to detect under strong competition. As seen above, this is the case in Drosophila melanogaster (Martín et al. 1988).

On the first day of egg harvest, the first series and second series of parents were used, respectively, to set up sib and random competition vials. The mating groups were returned to egg laying vials. In the following day, the use of the series was reversed, so that the first series was used to set up random competition vials, and the second series to set up sib competition vials.

It was necessary to ensure a minimum production of 100 eggs by each mating group used to set up sib competition vials. In order to increase egg production in this moment, each 20 -female group was divided into four five-female subgroups, and each subgroup was allocated to a separate vial.

Two weeks after the introduction of eggs, the number of larvae, pupae and adults per vial was scored. In every count, adults were discarded and the larvae 
and pupae returned to the vial. Weekly counts were made as long as living animals were found. Some competition vials lasted for 14 counts.

We used the SAS Generalized Linear Models procedure (SAS, 1982) to carry out all analysis of variance and covariance. As these were unbalanced, we used type III sums of squares in all cases.

\section{Results}

Despite the measures taken, some of the mating groups used to set up sib competition vials failed to lay 100 eggs, and the corresponding vials were started with fewer eggs. These vials were discarded for the analyses that follow, so that a total of 71 sib competition vials and 98 random competition vials were used.

\section{Mean week production}

The mean production of larvae and adults per week averaged over series and days is shown in Fig. 1. The average adult production per vial was 15.029 and 15.847 for the sib and random treatments, respectively.

A factorial analysis of variance including the effect of treatment (sibs and random), series of parents and day of setting up of the competition vials ( 1 and 2 ) was applied to each larvae, pupae and adults count (squareroot transformed). The treatment had a significant effect for larval counts $1\left(F_{1,165}=7.13, P<0.05\right), 2$ $\left(F_{1,164}=14.29, P<0.05\right), 6\left(F_{1,164}=5.86 \quad P<0.05\right), 7$ $\left(F_{1,164}=6.28, P<0.05\right)$ and $8\left(F_{1,162}=4.24, P<0.05\right)$ and in all these counts, the sibs treatment produced more larvae. There were no differences for any pupae or adults count. We applied the same analysis of variance model to study the total number of adults produced in each vial (this number was the sum of the

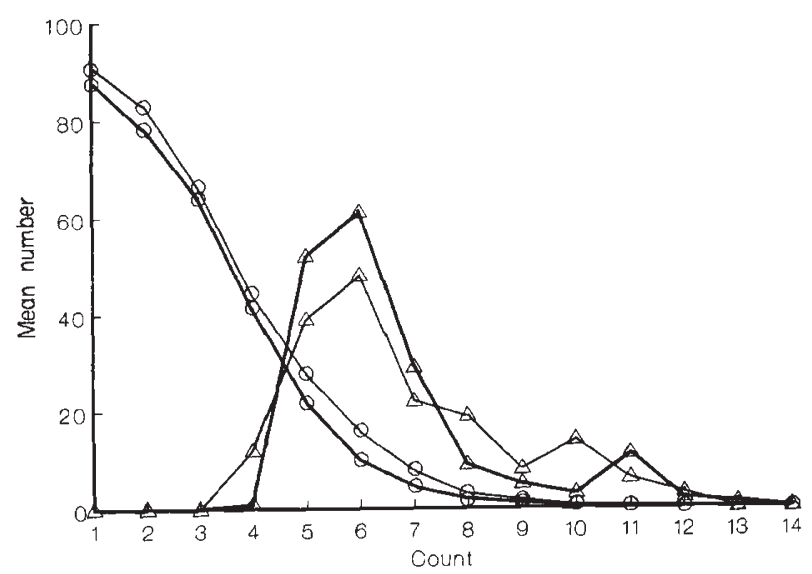

Fig. 1 Mean production of larvae $(0)$ and adults $(\Delta)$ for the sib (simple lines) and random (bold) competition vials. Adult numbers were multiplied by 10 . adult counts), and we did not find a significant effect for the treatment $\left(F_{1,164}=0.83, P>0.10\right)$. Thus, the greater larval production of the sibs treatment in the first weeks was not reflected in the greater total adult production.

We studied the effect of the larval density in the first weeks on total adult production per vial using an analysis of covariance model with the treatment, series of parents and day of setting up as factors, and the average larvae number for the first and second counts as a covariable. The interaction between the covariable and each factor was used to test for the homogeneity of the slope adjusted to the covariable for different levels of the factor.

The relationship between initial larval density and total adult production was negative in both treatments, but the difference in slope between treatments was clearly significant and therefore, the slopes were estimated separately for each treatment (Fig. 2). The adjusted slope for the random competition vials was steeper, and the proportion of the total variance for adult production that was explained by the model was greater also. Therefore, adult production was more density-dependent in the random vials.
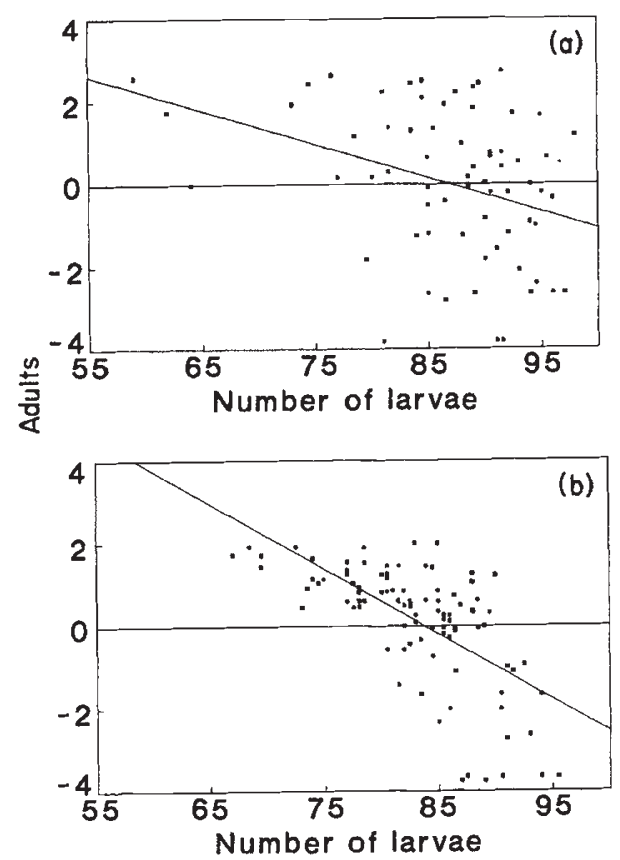

Fig. 2 The relation between total adult production per vial and initial larval density. Adult production was square-root transformed, and corrected for the effect of series of parents and day of setting up. (a) Sib-competition vials. (b) Random competition vials. The adjusted slopes were -0.082 $\left(t_{69}=-3.176, P<0.05\right)$ for $(a)$ and $-0.164\left(t_{95}=-8.803\right.$, $P<0.05)$ for $(b)$, and the $r$ square of the model was 0.128 for (a) and 0.449 for (b). 


\section{Larval viability}

The intensity of larval competition during the first weeks can be studied as the effect of larval density on larval viability. Viability was measured as the ratio of the number of larvae present in a given count to the number of larvae present in the previous count (arcsine transformed). An analysis of covariance model was used to analyse this viability. The effects considered were treatment, series of parents and day of setting up, and the larval density in the previous count was included as a covariable. The analysis was carried out only for the first to second count and second to third count viabilities, because many pupae began to appear in the fourth count, and the interpretation of larval viability was then complicated by the confusion of the effects of competition among larvae and larva-pupa cannibalism.

From the first to the second count, a clear difference between treatments for the effect of previous density (number of larvae in the first count) on larval viability was detected $\left(F_{1,160}=11.72, P<0.05\right)$. Thus, the slope for previous density was estimated separately for each treatment. It was negative and clearly significant $\left(b=-0.003, F_{1,93}=8.16, P<0.05\right)$ for the random treatment, showing that vials maintaining greater densities in the first counts tended to have greater mortalities in the following one. On the contrary, the slope for the sib competition vials was positive and nearly significant $\left(b=0.005, F_{1,67}=3.86, P<0.06\right)$.

In the second to third count viability, we did not find significant differences between treatments for the effect of previous density (second count density), and therefore, we used the data of both treatments to estimate the slope for this variable. It was positive and significant $\left(b=0.008, F_{1,119}=12.85, P<0.05\right)$, indicating either that vials maintaining higher previous densities had greater viabilities or, more likely, that the number of dead animals was both density independent and similar in all vials and, therefore, losses were proportionally lower where there were many larvae. This density independent viability (in the range of densities used in this experiment) could be ascribed to the procedure used to count the individuals in each vial. The culture medium was sifted and larvae and pupae were returned to the vial, this handling increasing mortality in crowded populations of Tribolium (Mertz \& Robertson, 1970).

\section{Larva-pupa cannibalism}

The viability of pupae in each count was estimated as the ratio of the number of adults found in that count to the number of pupae in the previous one (arcsine transformed). These viabilities were studied using an analy- sis of covariance model including treatment, series of parents and day of setting up as factors, and counts of larvae in the initial weeks as a covariable. The slope for previous larval density was positive and significant $\left(F_{1,68}=6.83, P<0.05\right)$ for the count 5 to count 6 viability, negative and significant $\left(F_{1,57}=26.58, P<0.05\right)$ for the count 6 to 7 viability, and non-significant for the rest.

However, no differences between treatments were found for mean viabilities or between the slopes adjusted to previous larval density and, therefore, there is not evidence of a reduced incidence of cannibalism among sibs.

\section{Inhibition of pupation}

At the end of the experiment, some vials maintained a rather stable group of big larvae in most counts, but failed to produce many adults. A posterior analysis showed that these vials were among those having the highest initial larval densities. We used an analysis of covariance model including treatment, series of parents and day of setting up as factors, and the average number of larvae in the first and second counts as a covariable. The dependent variable was the square root of the mean number of larvae found in each vial in the experiment's six final counts. The estimated slope for the covariable was $0.032\left(F_{1,159}=16.09, P<0.05\right)$. There was no significant difference in slope between treatments. Thus, our results indicate that larger larval densities in the first counts resulted in larger larval numbers in the final counts. The most likely explanation is that pupation was inhibited in vials having high initial densities, so that the proportion of individuals pupating in them was smaller, and the number of remaining larvae greater. Other cases of arrest in larval development have already been reported in crowded populations of Tribolium castaneum (Botella \& Mensua, 1986).

\section{Variances}

The between-vial variance for every larva, pupa and adult count was calculated separately for the sets of vials corresponding to the same treatment and series of parents, variances corresponding to the same treatment being pooled (Table 1). The between vial variance of total adult production was greater for the homogeneous treatment. The individuals in a homogeneous group were half and full-sibs, and an increase in between-group variance in this treatment indicates that the genetic origin of the vials had an effect on their production in competition conditions. This is in agreement with previous results in Tribolium castaneum (García \& 
Table 1 Between-vial variance for the number of larvae, pupae and adults found in each count. These variances were compared across treatments within counts using an $F$-test. '*' indicates the greater variance when significant differences were found at the 0.05 confidence level. The vials having a zero count were included in the calculation of the variances

\begin{tabular}{|c|c|c|c|c|c|c|}
\hline \multirow[b]{3}{*}{ Count } & \multicolumn{6}{|c|}{ Variance of the number of individuals } \\
\hline & \multicolumn{2}{|l|}{ Larvae } & \multicolumn{2}{|l|}{ Pupae } & \multicolumn{2}{|c|}{ Adults } \\
\hline & Sibs & Random & Sibs & Random & Sibs & Random \\
\hline 1 & 54.5 & 41.7 & 0.0 & 0.0 & 0.0 & 0.0 \\
\hline 2 & $73.6^{*}$ & 35.2 & 0.0 & 0.0 & 0.0 & 0.0 \\
\hline 3 & 93.4 & 68.4 & 0.0 & $0.1^{*}$ & 0.0 & 0.0 \\
\hline 4 & 459.5 & 312.5 & 85.6 & 75.1 & $35.8^{*}$ & 0.3 \\
\hline 5 & 442.2 & 371.3 & 69.9 & 48.7 & 76.8 & 91.1 \\
\hline 6 & 271.3 & 210.6 & 14.0 & $22.0^{*}$ & 69.9 & 56.1 \\
\hline 7 & $116.0^{*}$ & 78.4 & $13.3^{*}$ & 6.3 & 25.2 & 21.8 \\
\hline 8 & $43.2^{*}$ & 26.8 & $7.4^{*}$ & 3.2 & $16.3^{*}$ & 6.3 \\
\hline 9 & $22.1^{*}$ & 10.1 & $0.3^{*}$ & 0.1 & 3.9 & 3.1 \\
\hline 10 & $7.0^{*}$ & 1.9 & 0.1 & 0.1 & $0.4^{*}$ & 0.1 \\
\hline 11 & $2.4^{*}$ & 0.4 & $0.2^{*}$ & 0.0 & 0.1 & $0.4^{*}$ \\
\hline 12 & 0.1 & $0.3^{*}$ & 0.0 & 0.0 & 0.0 & 0.0 \\
\hline 13 & 0.0 & 0.2 & 0.0 & 0.0 & 0.0 & 0.0 \\
\hline 14 & 0.0 & 0.0 & 0.0 & 0.0 & 0.0 & 0.0 \\
\hline
\end{tabular}

Toro, 1990), showing that this between-vial genetic component had contributed to a positive response to group selection.

\section{Developmental time}

The day-of-pupation and day-of-adult-emergence can be estimated for each individual. An analysis of variance including as factors, treatment, series of parents and day of setting-up was applied to these data. No significant difference was found for day-ofpupation or day-of-emergence for any of the three factors.

As pupation day and emergence day were estimated for each individual in each vial, it was possible to split the total variance for these variables into betweenand within-vial components, and these can be compared across treatments. The between-vial variance for pupation day $\left(F_{45.49}=1.724, P<0.05\right)$ and day of emergence $\left(F_{44,49}=2.398, P<0.05\right)$ was larger in the sib than in the random treatment. This result indicates that the genetic origin of the individuals had an effect on their development time. Clear responses to artificial selection have been previously shown for this character (Dawson, 1967; Englert \& Bell, 1970; Soliman, 1982).

No differences between treatments were found for within-vial variance for pupation day $\left(F_{934,1253}=1.038\right.$, $\mathrm{ns})$, but this variance was greater in the random treatment for the day of emergence of adults $\left(F_{1007,1503}=1.282, P<0.05\right)$. The source of this difference can be identified when the variances are compared across variables within treatments. In the random treatment, we failed to detect significant differences when comparing the within-vial variances of pupation day and day of adult emergence $\left(F_{1503,1253}=1.037\right.$, ns $)$. However, the within-vial variance for pupation day was greater in the sibs treatment $\left(F_{934,1007}=1.282, P<0.05\right)$. Therefore, there was a reduction in within-vial variance in the sibs treatment in the pupa to adult step.

\section{Discussion}

In contrast with the predictions of the elbow-room model of sib competition, we have not detected a relationship between genetic homogeneity and total adult production.

In those groups genetically more homogeneous, we found a clear superiority in larval production in the first counts. These counts correspond to the second and third week of age of the individuals living in competition vials. This is an important period in the life-cycle of Tribolium, because pupation takes place in the third week for individuals grown in uncrowded situations (King \& Dawson, 1972), and pupae cannibalism by active forms is one of the most important factors regulating population size (Young, 1970).

The greater larval production found in the homogeneous groups was due to increased viability, because the number of eggs used in the sib and random treatments was the same. In addition, we found that viability was more dependent on density in the heterogeneous groups, and, as density dependence of viability is a clear indication of intraspecific competition (Keddy, 1989), we conclude that competition was less intense in the homogeneous groups in the first weeks. The elbow-room model assumes that competition intensity increases with genetic homogeneity, and, therefore, is not applicable when there is some kind of sib cooperation that may evolve through a process of kin selection.

Although no cases of co-operation among sibs had previously been detected in Tribolium, some mechanisms are known that could produce a fitness advantage for sib groups in this species. Karten (1965) compared the effects of conditioning the culture medium by wild type individuals and black mutants, and found that there is an advantage in oviposition rates for those individuals living in medium conditioned by animals of their own type. Fogle \& Englert (1976) observed that larvae of two strains of Tribolium preferred to eat eggs of the other strain, so that growth in number was lower in mixed populations. However, this was not fully con- 
firmed in posterior works (Mayes \& Englert, 1984). Wade (1980a) studied the cannibalistic behaviour of larvae randomly taken from a population of Tribolium, and did not find a preference for predation of eggs laid by non-relatives.

Jasienski et al. (1988) suggested a reduction in the behavioural antagonism among related individuals to explain a reduction in developmental time of sib groups of Tribolium confusum when compared with random groups of the same species. Our data seem to support this interpretation, as we observed a reduced density-dependence in larval mortality for the sib groups in the first counts in our experiment.

Individuals in the homogeneous groups could be more homogeneous for some characters, and this could also result in fitness advantages. By example, a reduction in variance in developmental time can result in a parallel reduction in the frequency of cannibalism (Dawson, 1975). When this variance is high, there are few pupae present in the population in a given time, and these pupae are surrounded by active and cannibalistic stages, as larvae and adults. As a consequence, pupa cannibalism is intense. In populations that pupate synchronically, pupae have greater viabilities because they meet a smaller number of individuals in active stages (Dawson, 1975). In our experiment, there was a reduction in developmental time variance from the pupation to the adult emergence in the sibs treatment, but this reduction was difficult to interpret, because it could be ascribed either to a reduction in the frequency of pupa cannibalism, due to a synchronization of adult emergence, or to an increase in the frequency of pupa cannibalism, resulting in the destruction of less synchronized pupae. Therefore, it could be related either to an advantage or a disadvantage for the vials in the sibs treatment. Had we found any difference in pupa viability between treatments, we would be able to resolve this difficulty, but, as seen above, it is not the case.

Whatever the mechanism responsible for the superiority in initial larval production in the sib groups, it failed to convert this superiority in superior adult production. This was due to the negative relationship between larval and adult production (Fig. 2). When the resources available are limited, as was the case with the culture medium in our experiment, they can be depleted before many individuals have finished their development. More crowded groups deplete their resources more quickly, and produce fewer adults (McDonald \& Davidson, 1965; Wade 1980b). In the homogeneous treatments, the advantage of having a reduced intensity of competition and larger population sizes in the first weeks was compensated in the long run by the disadvantage of a faster environmental deterioration, so that total adult production was similar in both treatments.

The between treatments difference in densitydependence found for total adult production needs some discussion. It might seem contradictory that the sibs treatment, which showed greater mortality from the first and second count to the adult stage, and therefore had an increased competition, had also a less density-dependent adult production. This apparent contradiction may be resolved by taking into account the effect of pupa cannibalism. Tribolium strains kept in the laboratory for many generations may maintain significant amounts of genetic variation for pupa cannibalism (Stevens, 1989), and this genetic variation should increase the between-groups variance for adult production in the sibs treatment. As this source of between-groups variance was not directly related to the larval densities in the first counts, the degree of density-dependence of the adult production in the sibs treatment was smaller than in the random treatment, in which the individuals in a group were randomly sampled from the population, and therefore the average pupa cannibalism in the groups was more similar. This interpretation is consistent with the greater variance for total adult production that was found in the sibs treatment.

The density-dependent regulation of adult production in our experiment could be mediated by increases in larval mortality, pupa cannibalism or pupation inhibition. All these mechanisms are densitydependent in Tribolium, but, to explain our results, a mechanism showing a clear density-dependence in the array of densities found in the competition vials is needed. This is not the case of larvae mortality in the first weeks, because it was density-dependent only for the first to second count of the random vials. On the other hand, pupa cannibalism was density-dependent only for one count. We think that it is the inhibition of pupation that could explain to a great extent why the vials maintaining more larvae produced less adults, because it was clearly density-dependent in both treatments. An alternative explanation for the positive relationship found between initial and final larval densities could be that the proportion of animals pupating and becoming adults is independent of initial larval density. Then, the number of remaining larvae would be greater in those vials having greater larval densities. But, as it has already been shown, these vials tended to produce a smaller number of adults rather than a greater one, as would be expected in principle. It could be argued that the proportion of individuals that pupate could be the same in all vials (so that there is not inhibition of pupation), but the viability of pupae is lower in those vials having higher initial larval densities, their adult produc- 
tion being therefore lower. But, as shown above, we failed to find differences in pupa viability between the sib and random treatments, that had different densities in the first weeks.

It is clear that our results are specific for the particular conditions considered. Had the competition intensity been reduced in the second week, i.e. by adding some fresh medium to the vials, the groups of sibs would have maintained their advantage up to the end of the experiment. On the other hand, had a certain amount of medium been removed in each vial, the resources available to produce adults would be scarcer in the sib groups, and fewer adults would be found in them. Thus, a positive relationship between genetic homogeneity in the groups and intensity of competition could be found, as expected in the elbow-room model. In this respect, Tonsor (1989) discussed the results found in his Plantago lanceolata experiment, and showed that modifications in its design could result either in the fulfilment of the elbow-room model's expectations, or produce opposite observations.

Our results illustrate the difficulties implied in the use of an ecological mechanism, as that included in the elbow-room model of sib competition, as a general explanation for the widespread presence of sexual reproduction. Such a mechanism must depend heavily on the peculiarities of the biology of each species and the environment in which each population lives. An enormous array of situations may be found among sexual reproducing organisms, and it is unlikely that a negative relationship between genetic similarity of neighbours and fitness could be found in all of them. Therefore, it is also unlikely that the elbow-room model can be of general validity.

\section{Acknowledgements}

We thank Carlos López-Fanjul for helpful comments on the manuscript, and Maribel Reyero and Leopoldo Sánchez for their help with the figures.

\section{References}

BARTON, N. H. AND POST, R. J. 1986. Sibling competition and the advantage of mixed families. J. Theoret. Biol., 120, $381-387$.

BOTELLA, L. M. AND MENSUA, J. L. 1986. Larval arrest in development of Tribolium castaneum (Coleoptera. Tenebrionidae). Environ. Entomol., 15, 1264-1267.

BULMER, M. G. 1980. The sib competition model for the maintenance of sex and recombination. J, Theoret. Biol., 82, $335-345$.

DAWSON, P. S. 1967. Developmental rate and competitive ability in Tribolium. II. Changes in competitive ability following further selection for developmental rate. Evolution, 21, 292-298.
DAWSON. P. S. 1975. Directional versus stabilizing selection for developmental time in natural and laboratory populations of four beetles. Genetics, 80, 773-783.

ELLSTRAND, N. C. AND ANTONOVICS. J. 1985. Experimental studies of the evolutionary significance of sexual reproduction. II. A test of the density-dependent selection hypothesis. Evolution, 39, 657-666.

ENGLERT, D. C. AND BELL, A. E. 1970. Selection for time of pupation in Tribolium castaneum. Genetics, 64, 541-552.

FOGLE, T. A. AND ENGLERT. D. C. 1976. Differential cannibalism among larvae of Tribolium castaneum as influenced by the antennapedia mutation. Can. J. Genet. Cytol., 18, 179-187.

GARCíA, C. AND TORO, M. A. 1990. Individual and group selection for productivity in Tribolium castaneum. Theor. Appl. Genet., 79, 256-260.

JASIENSKI, M. 1988. Kinship ecology of competition: size hierarchies in kin and non-kin laboratory cohorts of tadpolcs. Oecologia, 77, 407-413.

JASIENSK1, M., KORZENIAK, U. AND LOMNICK1, A. 1988. Ecology of kin and non-kin larval interactions in Tribolium beetles. Behav. Ecol. Sociobiol., 22, 277-284.

KARTEN, 1. 1965. Genetic differences and conditioning in Tribolium castaneum. Physiol. Zool., 38, 69-79.

KEDDY, P. A. 1989. Competition. Chapman \& Hall, London.

KELLEY, S. E. 1989a. Experimental studies of the evolutionary significance of sexual reproduction. V. A field test of the sib-competition lottery hypothesis. Evolution, 43, 10541065 .

KELLEY, S. E. 1989b. Experimental studies of the evolutionary significance of sexual reproduction. VI. Greenhouse test of the sib-competition hypothesis. Evolution, 43, 1066-1074.

KELLEY, S. E., ANTONOVICS, J. AND SCHMiTT, J. 1988. A test of the short-term advantage of sexual reproduction. Nature, 331, 714-716.

KING, C. F. AND DAWSON, P. s. 1972. Population biology and the Tribolium model. Evol. Biol., 5, 133-227.

MCDONALD, D. I. AND DAVIDSON, L. J. 1965. Population fitness in Tribolium. I. An approach to the problem. Am. Nat., 99, 463-470.

MARTIN, M. J., PéREZ-TOMÉ, J. M. AND TORO, M. A. 1988. Competition and genotypic variability in Drosophila melanogaster. Heredity, 60, 119-123.

MAYES, P. A. AND ENGLERT, D. W. 1984. Interstrain differences for larval dispersal and egg cannibalism in the flour beetle, Tribolium castaneum. Can. J. Genet. Cytol., 26, 420-424.

maynard Smith, J. 1978. The Evolution of Sex. Cambridge University Press, Cambridge.

MERTZ, D. B. AND ROBERTSON, J. R. 1970 . Some developmental consequences of handling, egg-eating and population density for flour beetle larvae. Ecology, 51, 989-998.

PÉREZ-TOMÉ, J. M. AND TORO, M. A. 1982. Competition of similar and non-similar genotypes. Nature, 229, 153-154.

PRICE, M. U. AND WASER, N. M. 1982. Population structure, frequency-dependent selection, and the maintenance of sexual reproduction. Evolution, 36, 35-43.

sAS. 1982. SAS User's Guide: Statistics. SAS Institute, Cary, N.C.

soliman, H. 1982. Directional and stabilizing selection for 
developmental time and correlated response in reproductive fitness in Tribolium castaneum. Theor. Appl. Genet., 63, 111-116.

STEVENS, L. 1989. The genetics and evolution of cannibalism in flour beetles (Genus Tribolium). Evolution, 43, 169-179.

TAYLOR, P. D. 1979. An analytical model of a short-term advantage for sex. J. Theoret. Biol., 81, 407-421.

TONSOR, S. J. 1989. Relatedness and intraspecific competition in Plantago lanceolata. Am. Nat., 134, 897-906.

WADE, M. J. 1980a. An experimental study of kin selection. Evolution, 34, 844-855.

WADE, M. J. 1980b. Group selection, population growth rate, and competitive ability in the flour beetle, Tribolium ssp. Ecology, 61, 1056-1064.

WILLIAMs, G. C. AND MITTON, J. B. 1973. Why reproduce sexually? J. Theoret. Biol., 39, 545-554.

WILLSON, M. F., THOMAS, P. A., HOPPES, W. G., KATUSIC-MALMBOURG, P. L., GOLDMAN, D. A. AND BothWELL, J. L. 1987. Sibling competition in plants: an experimental study. Am. Nat. 129, 304-311.

YOUNG, A. M. 1970. Predation and abundance in populations of flour beetles. Ecology, 51, 602-619.

YoUNG, J. P. W. 1981. Sib competition can favor sex in two ways. J. Theoret. Biol., 88, 755-756. 